\title{
Measurement of Visual Lobe Dimensions \\ with a Simultaneous Foveal Task
}

\author{
Alan H.S. Chan \\ Department of Manufacturing \\ Engineering \\ City University of Hong Kong \\ Hong Kong
}

\author{
Alan J. Courtney \\ Department of Industrial and \\ Manufacturing Systems Engineering \\ University of Hong Kong \\ Hong Kong
}

\begin{abstract}
This experiment studied the effects of different levels of foveal cognitive load on the dimensions of visual lobe. The dimensions of hard shell visual lobes were determined at the $50 \%$ detection threshold. Number of correct targets detected seemed unaffected by the foveal load in the near periphery but a decrement occurred beyond $7.7^{\circ}$, which indicates that there is no influence on the $50 \%$ hard-shell lobe value. But the tunnel vision found beyond this eccentricity decreases the detection probability of the soft-shell lobe. The target detection percentages obtained at no foveal load condition may give overestimates of detection probability for search condition with high foveal cognitive load
\end{abstract}

\section{INTRODUCTION}

Visual search has long been a topic for human factors studies in industry. In the electronics industry, recent studies have shown that identification of more than 160 potential types of rare defects by human inspectors is common in inspection of printed circuit boards [1]. It was reported studies in industrial inspection tasks ranged from microchip inspection [2] to aircraft inspection of cracks and corrosion. Reducing search time for computer menu displays and other visual display has been studied for typical human computer interaction tasks [3].

When we fixate a low-contrast target point in a uniform background we have maximum visual acuity along the line of sight and acuity decreases approximately linearly into the periphery, falling off more rapidly in the far periphery [4]. The limit of peripheral sensitivity for particular target and background characteristics in form detection tasks has been represented in terms of probability of target-acquisition as a function of eccentricity from fixation. The terms functional visual-field, visual lobe, and conspicuity area have been employed to describe the effective area within which a target would be seen with a given probability, e.g., $50 \%$ frequency of seeing.

Peripheral vision comprises most of the visual field and the co-operation of foveal and peripheral vision plays an important part in the total performance of human vision in man-machine systems. For instance, in supervisory tasks in a control room, aircraft control and car driving, the human operator usually needs to observe continuously and accurately several displays at the centre of his visual field. Simultaneously the operator has to detect and make response to the unpredictable alarm signals or unexpected events presented in the periphery of the visual field, which give vital information for the successful control of the machines and for survival. Fry [5] has stressed the importance of peripheral vision and its significant role in triggering subsequent eye movements with the available cues from peripheral vision. It is very time consuming and impractical to move the fovea to scan the entire field in a search task and so peripheral vision must be used in detection of a target in a search field.

It has been suggested that the extent and shape of the visual lobe is one of the main factors influencing overall search time in a particular task [6]. Bellamy and Courtney [7] found that there are large individual differences in lobe area even for people with comparable foveal acuity. Courtney and Chan [8] suggested that by careful choice of search material it may be possible to devise a search task to assess relative visual lobe size for screening individuals involved in search tasks, or to assess the effects of training. A relationship has been demonstrated between lobe area (or some related measures) and search performance for laboratory tasks [9] and for real targets with trained observers [10] and it is an important factor in mathematical modelling of visual search [11]. Some basic assumptions about the characteristics of visual lobes are usually made for modelling the visual search process e.g. circular or elliptical shapes but lobes may be more complex than this in practice.

In practice lobes have been found to be not at all circular and horizontal dimensions have been found to be significantly larger than vertical dimensions [12]. Extensive mapping of visual lobe areas showed that the boundaries were very irregular and there were regions of insensitivity within the lobe area, both of which may partly account for the difficulty experienced by some subjects in locating targets even after repeated scanning. These findings suggest that better knowledge of visual lobe area is needed for mathematical modelling and prediction of search performance.

In product inspection, an inspector normally has to decide whether the area around fixation contains a fault or not. The decision stage of inspection certainly involves a cognitive task performed at the fovea. In past studies, lobe size determination has mostly been performed using simple stimuli such as one static target on a homogeneous static background, with no foveal load processing during peripheral target detection. These considerations prompted the authors to initiate the study reported here on measurement of lobe dimensions with simultaneous processing of foveal load. If the foveal cognitive load interferes in any way with peripheral target detection, then visual lobe dimensions

0-7803-3280-6/96/\$5.00 $C_{1996 \text { IEEE }}$ - 2139 - 
obtained under conditions of no foveal load may overestimate lobe size and hence introduce inaccuracies in modelling for search performance prediction.

With the peripheral task as the primary task and the foveal task as secondary the experiment reported here investigated tunnel vision in this dual-task situation. Specifically the experiment aimed to find out if interference would be approximately equal at all eccentricities sampled, if there would be more interference as eccentricity increased, or if there is some combined effect of general interference and tunnel vision. The selective interference of tunnel vision, if it occurs, would be indicated by a significant interaction of foveal cognitive loads $x$ retinal eccentricities. Resources theory proposed by Norman and Bobrow [13] will be used as a framework to discuss any interference phenomenon observed in this dual-task situation by considering whether the tasks were in the resource-limited or data-limited region.

In the experiment reported here, only Arabic numerals were used in the foveal task and the number of numerals in each stimulus was kept constant at two for all levels of cognitive loading for the foveal task. Keeping the number of numerals constant was expected to eliminate any variations in visual complexity. Also this study examines whether the mere presence of a visual item requiring no response in the foveal region would lead to a decrement in dimensions of visual lobe.

\section{METHOD}

\section{Design}

In the experiment, the primary peripheral task was to locate the target (V) amongst the background characters (Xs) in the left and right meridians. There were four levels of secondary foveal task in this test. In the lowest Level 0 foveal task, there was no foveal load on the stimulus, and the subjects needed to perform the primary peripheral target detection only. In all Levels 1, 2 and 3 foveal tasks, foveal load was added onto the stimulus by placing a two-digit Arabic number at the centre of the stimulus. In Level 1 task, no response related to foveal load was necessary despite its presence. In Level 2 foveal task, the subjects were required to identify the foveal two-digit number. In the highest Level 3 task, the subjects were required to give the sum of the two digits in the number. A total of 128 presentations was tested for each of the four levels of foveal task giving a total of 512 trials for each subject. For each level, the 128 trials were divided into four sessions of 32 trials and a $2-\mathrm{min}$. rest was given between sessions. During each session of 32 trials, the target appeared twice at each of the 16 possible target positions. The order of presentation of the four levels of tasks was counterbalanced across the four sessions to reduce order effects.

\section{Stimuli}

Before and after a stimulus presentation, a fixation bracket "[]" was presented at the centre of the screen. The stimulus display passed through the fixation point and consisted of a horizontal row of 38 background character X's and one target character V. Characters were separated from each other by one character space width and the whole stimulus line was composed of a total of 78 characters and spaces. Two spaces appeared at the positions of the fixation bracket of stimulus line in the Level 0 task (Fig. 1a). Each of the stimulus lines for the Levels 1,2 and 3 tasks had a two-digit Arabic number which also appeared at the positions of the fixation bracket. One example of stimulus lines used in Levels 1,2 , and 3 tasks is shown in Fig, $1 \mathrm{~b}$. The foveal two-digit numbers were randomly selected from the range between 12 and 98 (excluding the same numeral numbers of $22,33 \ldots 88$ ). There were 19 characters on each side of the foveal number. Each stimulus line contained one target " $V$ " which appeared at one of 16 possible character positions (Fig. Ic) The eight eccentricities of target locations in the left and right fields were $2.2^{\circ}, 3.6^{\circ}, 50^{\circ}, 6.3^{\circ}, 7.7^{\circ}, 9.0^{\circ}, 10.4^{\circ}$, and $11.7^{\circ}$. The active viewing area of the stimulus line was $234 \times 45 \mathrm{~mm}$ presented at a distance of $500 \mathrm{~mm}$, subtending $2633^{\circ}$ horizontally and $31^{\prime}$ vertically to subjects eyes, Each target and background character was of $3 \times 4.5 \mathrm{~mm}$, subtending angles of 21 horizontally and 31 vertically with their centres separated by $6 \mathrm{~mm}\left(41^{\prime}\right)$. The two-digit number at the centre occupied two character spaces and was of $6 \times 4.5 \mathrm{~mm}$, subtending angles of $41^{\prime}$ horizontally and $31^{\prime}$ vertically $\mathrm{A}$ postexposure masking line (Fig ld) with all the background and target positions filled with asterisks (*) was presented immediately after the stimulus. The luminance of the background characters, target, and foveal number was 16.7 $\mathrm{cd} / \mathrm{m}^{2}$ and the background luminance was about $3.0 \mathrm{~cd} / \mathrm{m}^{2}$.

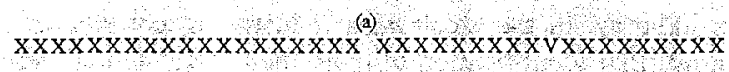

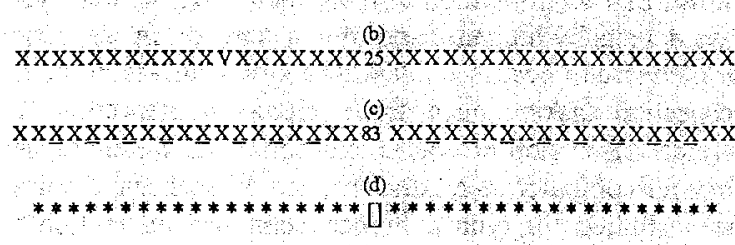

Fig. 1

(a) An example of a stimulus line in Level 0 task One target $V$ appeared amongst background characters of Xs.

(b) An example of a stimulus line in Level 1, Level 2 and Level 3 tasks. One target $V$ appeared amongst background characters Xs. A two-digit Arabic number appeared at the centre.

(c) Underlined are the 16 possible target positions on foveal stimulus line.

(d) The postexposure masking line (not to scale).

\section{Subjects}

Three female and five male subjects without previous experience on the tasks participated in this experiment. Their ages ranged from 20 to 23 years, with median of 22 All subjects had near acuity scores of at least 8 (20/25 Snellen notation) on the Bausch and Lomb Orthorator. 


\section{Apparatus}

A personal computer was used in this experiment for presentation of the centre fixation bracket and stimulus lines. A standard numeric keyboard was used by subjects for controlling stimulus presentation and for inputting peripheral and foveal responses. The "Enter" key was for controlling stimulus presentation and target position confirmation. The "Left Arrow" and "Right Arrow" keys were used for indicating the side (left or right) on which the peripheral target was detected. These two arrow keys were also used by subjects for moving the cursor to the left or right on the 38 character positions along the stimulus line for estimation of target location. The numeric keys from 0 to 9 were used for responses to the foveal task. An adjustable chair was used for subjects' comfort and to ensure that line of regard was roughly perpendicular to and at the centre of the screen.

\section{Procedure}

Near acuity was tested before the experiment for each subject. At the beginning of each session, eight practice trials randomly selected from the 32 stimuli of that session were given to familiarise subjects with use of the numeric key-pad and the overall procedure. It also allowed subjects to become accustomed to thinking of the peripheral task as the primary and the foveal task as the secondary task. Each stimulus line was presented for $250 \mathrm{msec}$. after initiation by subjects. This duration was short enough to minimise saccadic movements during stimulation in view of uncertainty of target locations in the test and was used previously in related experiments [14][15]. The subjects fixated the centre bracket "[]" before they were ready for the initiation of stimulus presentation. Immediately after each presentation, the masking line with *s on previous Xs and target positions was displayed and the subject was required to move the cursor from the centre with the "Left Arrow" or "Right Arrow" key to their estimate of target position and pressed the "ENTER" button for confirmation of target position. On both the Level 0 and Level 1 tasks, no response for the foveal stimulus was needed. On the Level 2 task, subjects had, subsequent to response to the primary task, to press two appropriate buttons on the numeric pad indicating the numbers they saw at the centre and then pressed "Enter" for confirmation. On the Level 3 task the subjects had to input the sum of the two digits of the foveal number instead of the number itself. With this input procedure, subjects were forced to give response to the primary peripheral task first and secondary foveal task later. No feedback was given to subjects.

\section{RESULTS}

\section{Peripheral Task}

For the peripheral task, the distance between the actual and estimated target position was taken as the error score for a response. A response was correct when the error score was no greater than two character locations (i.e., about $1^{\circ} 22^{\prime}$ ). The number of correct responses made at the four levels represents the extent of peripheral target detection of subjects. Over all of the 512 presentations, the best subject had a total of $376(73.4 \%)$ correct responses and the poorest one with $210(41.0 \%)$. Student's $t$ test performed on the number of correct responses made at each eccentricity showed that there was no significant difference between leftand right-field sensitivity for all four levels of foveal task ( $p$ $>0.1$ ), which justified the pooling of left and right correct numbers for each level in further analysis.

In Fig. 2, the four pooled data curves (one for each foveal load) overlap each other from $2.2^{\circ}$ to $7.7^{\circ}$, then diverge. The Level 0 curve is at the top and the Level 3 curve is at the bottom. Levels 1 and 2 curves are in between and very similar to each other. For each of the four task levels, oneway analysis of variance of the number of correct responses across the four sessions showed that practice effects were nonsignificant $(p>.1)$. Since each subject only performed the test under one of the four different orders of presentation, the experiment was conducted under a crossed and nested design or nested-factorial design. Subjects were nested within orders, whereas levels and orders were crossed.

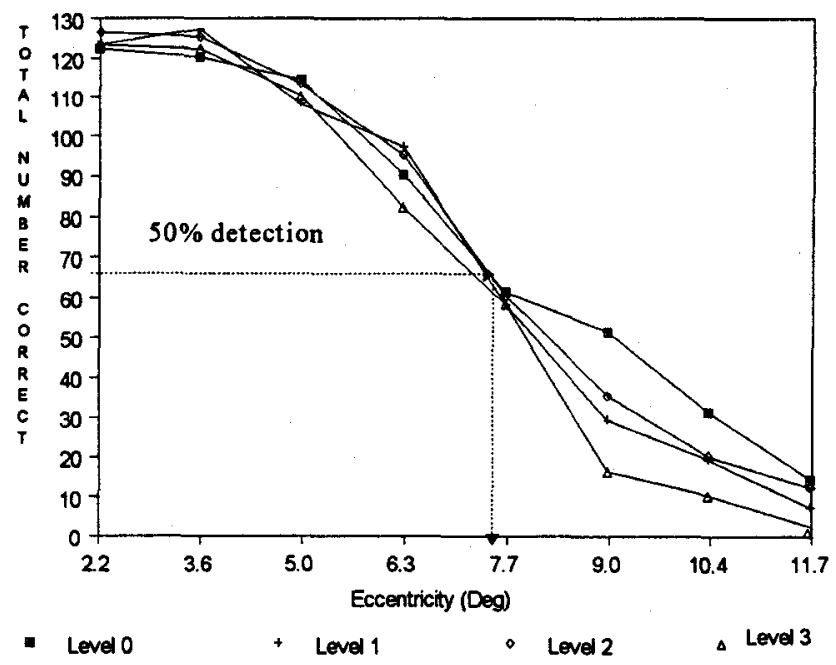

Fig. 2 Total number of left and right pooled, correct peripheral responses vs eccentricity.

A complete analysis of variance with this design showed that levels was significant $\left(F_{3,12}=6.08, p<.01\right)$ and subjects within orders also differed significantly $\left(F_{4,12}=22.65, p<\right.$ $.001)$. Orders of presentation was a nonsignificant effect $(p>$ .1). There was also a significant interaction between levels and orders $\left(F_{9,12}=16.21, p<.001\right)$ and a nonsignificant interaction $(p>.1)$ between levels and subjects within orders.

The experiment was also considered as a three-factor factorial design with main factors of eccentricities, levels and subjects. Analysis of variance showed that eccentricities was a significant factor $\left(F_{7,256}=379.56, p<.001\right)$, indicating that the number of correct responses varied significantly at different eccentricities. The levels factor was also significant $\left(F_{3,256}=6.31, p<.01\right)$ which showed that subjects performed significantly differently across the four levels. Performance between subjects also differed significantly $\left(F_{7,256}=32.41, p\right.$ $<.001)$. The computed $F$ ratio of 1.51 for the interaction between eccentricities and levels was marginally smaller 
than the critical value of $1.56\left(F_{21,256, p}=.05\right)$, indicating mild evidence for tunnel vision in this experiment. Significant interaction was also found between levels and subjects $\left(F_{21,256}=7.81, p<.001\right)$, showing that subjects' relative performance across the four levels of task were different. The interaction between eccentricities and subjects was also significant $\left(F_{49,256}=4.86, p<001\right)$. The three-factor interaction of subjects, levels and eccentricities was insignificant $(p>.01)$.

\section{Foveal Task}

The accuracy of identification and summation of the two centre foveal numerals was taken as the performance measure for Level 2 and Level 3 foveal task, respectively. Subjects made a total of $903(88.18 \%)$ correct identifications and $838(81.84 \%)$ correct summations in Level 2 and Level 3 tasks, respectively. However, for each of the Levels 2 and 3 tasks, student's t tests showed that there was no significant difference $(p>.1)$ between the numbers of correct foveal responses when the peripheral target was in the left or the right field, suggesting the grouping of the numbers correct for each eccentricity from left and right fields.

One-way analysis of variance on the temporal pattern of the responses indicated no significant variation in the number of correct foveal responses made at the beginning and at the end of the testing sessions in both Level $2\left(\mathrm{~F}_{3,31}=0.46, p>.1\right)$ and Level 3 tasks $\left(\mathrm{F}_{3,31}=0.26, p>.1\right)$.

As shown in Fig. 3, the Level 2 foveal task performance did not vary too much with eccentricity. Regression correlation for the performance function line was .087 , showing that very small variation in correct number was accounted for by variation of eccentricities. Nevertheless, analysis of variance for test for lack of fit showed that a linear model was adequate $(p<.001)$. The Level 3 curve dropped with increase of eccentricity, and the regression coefficient was -0.757 ( $p<$ .025). A test for lack of fit also showed that the regression line was linear $(p<.001)$. A three-factor analysis of variance was performed on the number of correct foveal responses with main effects of eccentricities, levels and subjects. All the three main factors of eccentricities $\left(F_{7,128}=2.85, p<.01\right)$, levels $\left(F_{1,128}=20.61, p<.001\right)$, and subjects $\left(F_{7,128}=17.27\right.$, $p<.001)$ were significant. Amongst the two-factor interactions, only levels $\mathrm{X}$ subjects was significant $\left(F_{7,128}=\right.$ 21.91, $p<001)$. The three-factor interaction was nonsignificant $(p>.5)$.

\section{Correlation Between Peripheral Task and Foveal Task Performance}

No significant correlations were found between subjects' foveal task performance and peripheral task performance in Level 2 (regression coefficient, $r=0.440$; Spearman's rank correlation coefficient, $\left.r_{s}=0.149\right)$ and Level $3\left(r=0.292, r_{s}=\right.$ -0.435 ) task. This showed that subjects who were good in the primary peripheral task might be good or bad in the secondary foveal task.

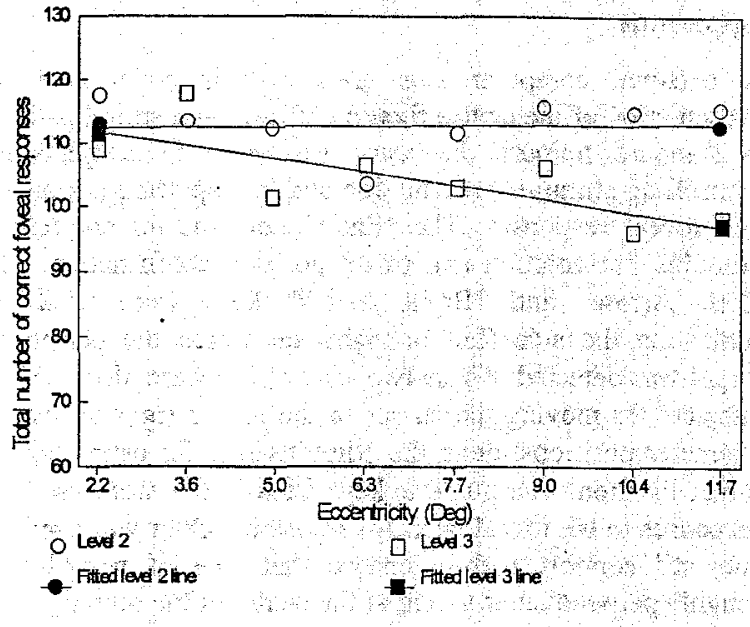

Fig. 3 Total number of correct foveal responses $v$ eccentricity.

\section{DISCUSSION}

This experiment measured visual lobe dimensions of subjects with simultaneous processing of various levels of foveal cognitive load. The current peripheral task was detection of a target against a competing background and the foveal task here occupied 41 ' of the centre region and was practically a static numeral identification or summation task.

\section{Peripheral Task}

The $41.0 \%$ to $73.4 \%$ range of conect peripheral responses made by subjects showed that the primary task was a good test of discrimination, being neither too difficult nor too easy. The left-and right-field sensitivity limits were statistically the same for each of the four levels of foveal task, agreeing with the results found in previous researches on visual lobe characteristics with no foveal load [14] and showed that there is no left-right hemispheric asymmetry in this task.

The overall $95.7 \%$ correctness for the four levels of task at $2.2^{\circ}$ and $3.6^{\circ}$ showed that subjects detected targets at these two eccentricities with very high probability of success, missing only $4.3 \%$ of targets appearing on the parafoveal region, prebably because of loss of concentration. All subjects had correct locations at these two eccentricities for all four levels of foveal task. Even the poorest subject had two and four correct locations at the nearest target locations in the right and left fields, respectively, for the most difficult foveal task. No subject obtained a correct location for the $11.7^{\circ}$ eccentricity and the observed $6.1 \%$ correctness at this eccentricity was close to the probability of a correct guess of $5.3 \%$. The lack of any practice effects found for the peripheral task reflected the consistency of performance across the four sessions and indicated that the 512 presentations could be reduced to shorten the test time without losing much accuracy or reliability.

Analysis of variance based on nested factorlal design indicated that level of foveal loading significantly affected performance on the peripheral task. A significant effect for subjects showed that individuals tested with same order of levels differed in performance while a nonsignificant order effect showed that the number of correct responses had no 
specific relation with the order of presentation of the four levels of foveal task. A significant interaction between levels and orders indicated that subjects' relative performance on the four levels of task were different for the four different orders of presentation. Subjects' relative performance depended not only on level but also on its interaction with the order of presentation. This interaction was not interpreted as due to practice nor to order of presentation which had already been found nonsignificant but was derived from inconsistent pattern of relative performance of individuals across the four levels. The nonsignificant interaction between levels and subjects within orders indicated that, although levels ard orders interacted, subjects under a specific order of presentation had similar patterns of results across the four levels. Similar to the results obtained in the nested-factorial design, the three-factor analysis of variance showed that the three main factors of eccentricities, levels, and subjects were all significant.

\section{Foveal Task}

The high percentages of correct responses for the foveal task at Levels 2 and 3 showed that they were relatively easy. The overall accuracy of identification and summation of central numerals made by all subjects were shown not to be affected by whether the peripheral target was in the left or the right field. Test for lack of fit for data in Fig. 3 showed that the total number of correct responses for the foveal task made on Levels 2 and 3 tasks could be described by linear models. No variation of performance with eccentricity was found on the Level 2 task while performance dropped across eccentricities on the Level 3 task. The further the peripheral target was away from the centre, the worse the performance was on the Level 3 foveal task. This suggested that sufficient resources were allocated for Level 2 foveal task but not for Level 3. The unchanged performance on the Level 2 foveal task across all eccentricities suggested that it probably had higher priority in capturing resources from the resource pool although subjects were told that it was the secondary task. No performance decrement was observed and a flat performance curve resulted while the primary peripheral task showed performance decrement at eccentricities beyond $7.7^{\circ}$. No changes in performance of the central task were noted in the study. With the resources theory, the result here indicates that the Level 2 foveal task was in the data-limited region. The level of resources where performance becomes datalimited, $\mathrm{R}_{\mathrm{dl}}$ for the foveal task was so low that it was smaller than the resources allocated for the foveal task even when the peripheral target was at $11.7^{\circ}$. For the Level 3 task, the observed performance decrement showed that it was resource-limited and the $\mathrm{R}_{\mathrm{dl}}$ was higher than the level of resources allocated for the task even when the target was at $2.2^{\circ}$.

\section{Interference}

The four overlapping curves for different levels of foveal task in Fig. 2 showed that at the near periphery the foveal loads did not interfere much with the peripheral task when the target was within $7.7^{\circ}$. Within $7.7^{\circ}$ the peripheral task either was easy enough to leave surplus resources for the processing of the foveal cognitive load or there was enough time to do them both. As the attentional resources for performing concurrent tasks is limited and the two tasks compete for a common and fixed supply of resources, it is reasonable to assume that the allocation of resources to them is reciprocal. When the target was located at greater eccentricities, the secondary task was sufficiently difficult to affect the primary one. As shown in Fig. 2, Level 1 performance was relatively poorer than Level 0 beyond $7.7^{\circ}$, showing that the mere presence of foveal numerals caused a performance decrement beyond an eccentricity of $7.7^{\circ}$. This suggested that there was competition between these two time-shared tasks for resources at eccentricities greater than $7.7^{\circ}$. Levels 1 and 2 did not differ much beyond $7.7^{\circ}$, suggesting that the identification task performed at the centre required few additional resources. This suggests that peripheral target detection is not affected when the foveal task is relatively easy. It appears that identification of numbers is so highly practised an activity that it becomes automatic and thereby requires minimal attentional resources. Another possibility was that because these numerals were presented at the centre of fixation, they were attended to at Level 1 as at Level 2 although the subjects were not asked to respond to them at Level 1. Leibowitz [16] reported that "a large number of studies illustrated the principle that the effect of peripherally presented stimuli is variable. A generalisation emerging from these data is that, when foveally and peripherally presented stimuli compete for attention, the peripheral stimuli have lower priority."

Performance on the primary peripheral task with the central secondary Level 3 task deteriorated beyond $7.7^{\circ}$, showing a steeper slope than with Levels 1 and 2 (Fig. 2), and thereby constituting evidence of the tunnel-vision effect suggested by the analysis of variance.

For the Level 2 foveal task, it appears that the resources allocated for the peripheral task were less than its $R_{d l l}$, it was resource-limited in the range of eccentricities tested, and performance was affected by competition for resources with the foveal task. Norman and Bobrow [13] stated that "the asymmetry of interference between two tasks is likely to depend in large part upon task instructions and subject strategy - upon which of the competing tasks receives first priority. The high priority will tend to be data-limited and the low-priority task resource-limited." This suggested that the data-limited Level 2 foveal task was the one processed with higher priority under conscious or unconscious conditions, most likely based on subjects' strategy, though subjects were instructed to think it was the secondary task. Compared with subjects' strategy, task instructions seemed to have comparatively smaller effect on the priority of tasks in this test.

For the Level 3 task on which both primary and secondary tasks showed performance decrements with increasing eccentricity, the results suggested that competition for resources by the two time-shared tasks did occur. Even with the advantage of being central, the foveal task was sufficiently difficult and resource demanding that its performance could not be maintained across eccentricities and it was resource-limited. The number of correct foveal responses dropped at a rate of 1.6 per degree. As performance on both the peripheral and foveal tasks were affected, they were both in the resource-limited region within 
the range $2.2^{\circ}$ to $10.4^{\circ}$. The small number of comect peripheral responses beyond $10.4^{\circ}$ indicated that the peripheral task became data-limited thereafter.

If a $50 \%$ target detection is taken for determining the limit of sensitivity for a hard-shell lobe in Fig. 2, the tunnel vision observed does not change this limit. But the tunnel vision found beyond $7.7^{\circ}$ decreases the detection probability of the soft-shell lobe[17] [18] beyond this eccentricity. Peripheral task performance of subjects will then be reduced if the secondary foveal cognitive task is sufficiently difficult and takes much resources from the primary peripheral task. This may happen when the potential fault in the foveal region is difficult to classify and more attentional resources are drawn from the peripheral task. An implication of this phenomenon is that visual field dimensions are not static during visual search, but change momentarily with the difficulty of foveal task at each fixation, depending on whether the details in the foveal region depletes resources from resource-limited peripheral processing or not. Or, in a real search task, duration of fixation is unconsciously lengthened for increased processing requirements as required by the dual tasks. This hypothesis can be validated by measuring visual lobes with foveal load with a self-paced presentation time.

\section{SUMMARY}

This experiment investigated the effects of foveal cognitive load on a the dimensions of visual lobe. At a low cognitive load, foveal task performance showed no deterioration for all eccentricities tested, but at a higher cognitive load, performance declined gradually across eccentricities. Mild evidence of tunnel vision was indicated by the significant interaction of cognitive loads $\mathrm{x}$ eccentricities. Resources theory accounted well for the results Number of correct targets detected seemed unaffected by the foveal load in the near periphery but a decrement occurred beyond $7.7^{\circ}$, which indicates that there is no influence on the $50 \%$ hard-shell lobe value, but the target detection percentages obtained at no foveal load condition may give overestimates of detection probability for search condition with high foveal cognitive load. This happens when an inspector is required to process peripheral information within the single target lobe while at the same time trying to determine whether a potential fault in the foveal region really constitutes a fault or not. Under such a condition, wrong estimate of lobe size may give erroneous results if a soft shell lobe model is used in mathematical modelling for prediction of visual search performance.

\section{REFERENCES}

[1] Stewart, J.R, 1990, Operator performance predictability in electronic circuit board inspection, unpublished $\mathrm{PhB}$ thesis, Texas A \& M University.

[2] Bhatnager, V.W., 1988, The effects of experience on search strategy, performance and stress during microscopic inspection of real IC chips, unpublished PhD thesis, State University of New York at Buffalo.
[3] Scott, D and Findlay, J.M, 1990, Visual search and VDUs, in Visual Search 2 by D. Brogan, A. Gale and K, Carr (eds) (Taylor \& Francis, London), 301-307.

[4] Bloomfield, J.R., 1975, Theoretical approaches to visual search, in Human reliability and quality control by J.G. Fox and C.G. Drury (eds) (Taylor \& Francis, London), 19-29.

[5] Fry, S.G., 1960, Visual Search Techniques by A Morris and E.P. Home (ed), Amed Forees NRC Committee on Vision, NAS-NRC, Washington, D.C., 233

[6]Kraiss, KF. and Knaeuper, A, 1982, Using visual lobe area measurements to predict visual search performance, Human Factors, 24, 673-682.

[7] Bellamy, L.J. and Courtney, A.J, 1981 , Development of a search task for the measurement of periplieral visual acuity, Ergonomics, 24(7), 497-509.

[8] Courtney, A.J. and Chan, H.S., 1986, Manual card sorting compared to automatic card presentation for assessing visual lobe size, Ergonomics, 29, 1553-1560.

[9] Johnston, D.M., 1965, Search performance as a function of peripheral acuity, Human Factors, 7, 537-551

[10] Leachtenauer, J.C, 1978 , Peripheral acuity and photointerpretation performance, Human Factors, 20, 537-551.

[11] Overington, I, 1979, The current status of mathematical modelling of threshold functions, in Search and the human observer by JN. Clare and M.A. Sinclair (eds) (London: Taylor and Francis), 14-125.

[12] Courtney, A.J and Chan, HS, 1985, Visual lobe area for single targets on a competing homogeneous background, Human Factors, 27(6), 643-652.

[13] Norman, D.A and Bobrow, D.G, 1975, On data-limited and resource-limited processes, Cognitive Psychology, 7, 4464.

[14] Courtney, A.J and Chan, H.S., 1985, Simple measures of visual-lobe size and search perfomanee, Ergonomics, 28 , 1319-1331.

[15] Courtney, A.J and Chan, H.S., 1986, Visual lobe dimensions and search performance for targets on a competing homogeneous background, Perception \& Psychophysics, 40(1), 39-44.

[16] Leibowitz, H.W, 1973, Detection of peripheral stimuli under psychological and physiological stress, in Visual search by Nationat Research Council Committee on Vision (ed), Washington, DC National Academy of Seiences, 6476 .

[17] Brown, M.B., 1979, Mathematical approaches to modelling visual search, in Search and the human observer by J.N. Clare and MA Sinclair (eds) (Taylor and Francis, London), 126-131.

[18] Bowler, YM, 1988, Towards a simplified model of visual search, in Visual Search by D Brogan (eds) (Taylor \& Francis, London), 303-309. 